\title{
An Imperialist Competitive Algorithm-Artificial Neural Network Method to Predict Oil Flow Rate of the Wells
}

\author{
Shahram Mollaiy Berneti \\ Automation and Instrumentation Department \\ Petroleum University of Technology \\ Ahwaz, Iran
}

\author{
Mehdi Shahbazian \\ Automation and Instrumentation Department \\ Petroleum University of Technology \\ Ahwaz, Iran
}

\begin{abstract}
Flow rates of oil, gas and water are most important parameters of oil production that is detected by Multiphase Flow Meters (MFM). Conventional MFM collects data on long-term, because of the radioactive source is used for detection and in unmanned location used due to being away from wells. In this work, a new method based on feed-forward artificial neural network (ANN) and Imperialist Competitive Algorithm (ICA) have been proposed to predict of oil flow rate of the wells. The proposed algorithm combines the local searching ability of the gradientbased back-propagation (BP) strategy with the global searching ability of imperialist competitive algorithm. Imperialist Competitive Algorithm is used to decide the initial weights of the neural network. The ICA-ANN is applied to predict oil flow rate of the wells utilizing data set of 31 wells in one of the northern Persian Gulf oil fields of Iran. The performance of the ICA-ANN is compared with ANN and the results demonstrate the effectiveness of the ICA-ANN.
\end{abstract}

\section{Keywords}

Artificial Neural Network, Back-Propagation, Oil Flow Rate, Multiphase Flow meter, Imperialist Competitive Algorithm

\section{INTRODUCTION}

Flow rates, temperature and pressure are important parameters of oil production that effect on production forecast and reservoir simulation. Temperature and pressure of each well are measured by digital sensors and flow rates are measured by Multiphase Flow Meter (MFM). MFM is one of the key technologies to improve production confidently which measures unseparated oil, gas and water flow rates; But Most of them have several technical problems. Some of them have radioactive source, controlled and checked manually, long distance between wells, discreet data gathering and high facilities cost maintenance. Operational person, environmental, time and cost problems require to use more quick and cheap alternative ways.

Artificial neural network (ANN) is a conceptual device whose output or inferred variable can be modeled in terms of other parameters that are relevant to the same process [1]. According to Rallo et al., artificial neural network could be used for prediction of the oil flow rate of the wells.

The determination of network structure and parameters are very important. Some evolutionary algorithms such as Genetic Algorithm (GA) [2], Simulated Annealing [3], Pruning Algorithm [4] and Particle Swarm Optimization [5] can be used for this determination. Recently, a new evolutionary algorithm has been proposed by Atashpaz-Gargari and Lucas [6], which has inspired from a socio-political evolution, called Imperialist
Competitive Algorithm (ICA). This evolutionary optimization strategy has shown great performance in both convergence rate and better global optima achievement [7], [8], [9], [10].

In the present work, we propose ICA for optimizing the initial weights of feed-forward neural network. The simulation results demonstrate the effectiveness and potential of the new proposed network for oil flow rate prediction in one of the northern Persian Gulf oil fields of Iran compared with BP neural network using the same observed data.

\section{ARTIFICIAL NEURAL NETWORKS}

Artificial neural networks is a parallel distributed processor which can express complex and nonlinear relationship, by using input-output training patterns from the experimental data. ANNs provides a non-linear mapping between inputs and outputs by its intrinsic ability [11].

The most common neural network architecture is the feedforward neural network with the Back-Propagation (BP) training algorithm. Feed-forward network is the network structure in which the information or signals will propagates only in one direction, from input to output. A three layered feed-forward neural network with back propagation algorithm can approximate any nonlinear continuous function to an arbitrary accuracy [12].

The network is trained by performing optimization of weights for each node interconnection and bias terms; until the values output at the output layer neurons are as close as possible to the actual outputs. The mean squared error of the network (MSE) is defined as:

$$
M S E=\frac{1}{2} \sum_{k=1}^{G} \sum_{j=1}^{m}\left[Y_{j}(k)-T_{j}(k)\right]^{2}
$$

where $m$ is the number of output nodes, $G$ is the number of training samples, $Y_{j}(k)$ is the expected output, and $T_{j}(k)$ is the actual output. The data are split into two sets, a training data set and a validating data set. The model parameters are estimated using only the training data. The validating data are used to estimate the accuracy of the model performance.

BP is a gradient descent algorithm on the error space, which most likely gets trapped into a local minimum, making it entirely dependent on initial (weight) settings. This shortcoming can be removed by global searching ability of the evolutionary algorithms such as ICA. 


\section{IMPERIALIST ALGORITHM}

The Imperialist Competitive Algorithm (ICA) is a new evolutionary algorithm in the Evolutionary Computation field based on the imperialistic competition [6]. Like other evolutionary algorithms the ICA starts with initial populations called countries $\left(N_{\text {country }}\right)$. There are two types of countries; colony $\left(N_{\text {col }}\right)$ and imperialist $\left(N_{\text {imp }}\right)$ (in optimization terminology, countries with the least cost) which together form empires. The initial number of colonies of an empire is convenience with their powers. To divide the colonies among imperialists proportionally, the normalized cost of an imperialist is defined by:

$$
C_{n}=c_{n}-\max _{i}\left\{c_{i}\right\}
$$

where $C_{n}$ is the cost of nth imperialist and $C_{n}$ is its normalized cost. Having the normalized cost of all imperialist, the power of each imperialist is calculated by:

$$
P_{n}=\left|\frac{C_{n}}{\sum_{i=1}^{N_{i m p}} C_{i}}\right|
$$

After forming initial empires, the colonies start moving toward their relevant imperialist country. Figure 1 [1] shows this movement. In this figure the colony moves toward the imperialist by $\mathcal{X}$ (is a random variable with uniform distribution) units.

$$
x \sim U(0, \beta \times d)
$$

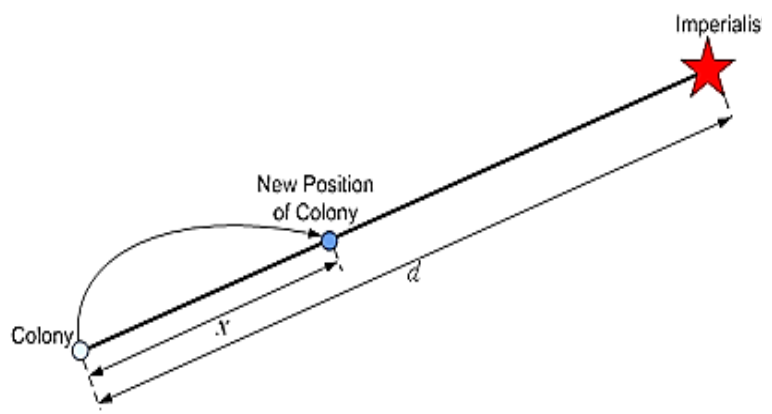

\section{Fig 1: Movement of colonies toward their relevant imperialist}

where $\beta$ is a number greater than 1 and $\mathrm{d}$ is the distance between the colony and the imperialist. The total power of each empire depends on both the power of the imperialist country and the power of its colonies. This fact is modelled by defining the total cost by:

$$
\begin{aligned}
\text { T. } C_{\cdot n}=\operatorname{Cost}\left(\text { imperialist }_{n}\right) & \\
& +\xi \operatorname{mean}\left\{\operatorname{cost}\left(\text { colonies of impire } e_{n}\right)\right\}
\end{aligned}
$$

where T.C.n is the total cost of the $n$th empire, and $\xi$ is a positive number which is considered to be less than 1 . A little value for $\xi$ causes the total power of the empire to be determined by just the imperialist and increasing it will increase the role of the colonies in determining the total power of an empire.
In the imperialistic competition process, imperialists try to attempt to achieve more colonies. So during the competition the powerful imperialists will be increased in the power and the weak ones will be decreased in the power. When an empire loses all of its colonies, it is assumed to be collapsed. At the end the most powerful imperialist will remain in the world and all the countries are colonies of this unique empire. In this stage the imperialist and colonies have the same position and power.

\section{ICA-ANN NETWORK RESULTS}

In this study, an Imperialist Competitive Algorithm-Artificial Neural Network (ICA-ANN) method was used to predict the oil flow rate of the wells by using the data set of 31 wells in one of the northern Persian Gulf oil fields of Iran. Data set are collected in three month periods for each well from Dec.2002 to Nov.2010. The best ANN architecture was: 2-7-1 (2 input units, 7 hidden neurons, 1 output neuron). ANN model trained with back propagation (Levenberg-Marquardt) using two parameters (temperature, pressure) as inputs. The transfer functions in hidden and output layers are sigmoid and linear, respectively.

ICA is used as neural network weight initialization algorithm and The Mean Square Error (MSE) used as a cost function in this algorithm. The goal in the proposed algorithm is minimizing this cost function. In these simulations, the number of imperialists and the colonies are considered 4 and 40, respectively; parameter $\beta$ is set to 2 . The number of training and testing data is 400 and 200, respectively.

The simulation performance of the ICA-ANN model was evaluated on the basis of mean square error (MSE) and efficiency coefficient $R^{2}$ [13]. Table 1 gives the MSE and $R^{2}$ values for the two different models of the validation phases. It can be observed that the performance of ICA-ANN is better than ANN. Figures 2, 3, 4 and 5 shows the comparison between predicted and measured normalized oil flow rate of the wells at training and validation phases for ICA-ANN and ANN models, respectively. Figures $6,7,8$ and 9 show the extent of the match between the measured and predicted oil flow rate of the wells at training and validation phases by ICA-ANN and ANN models in terms of a scatter diagram.

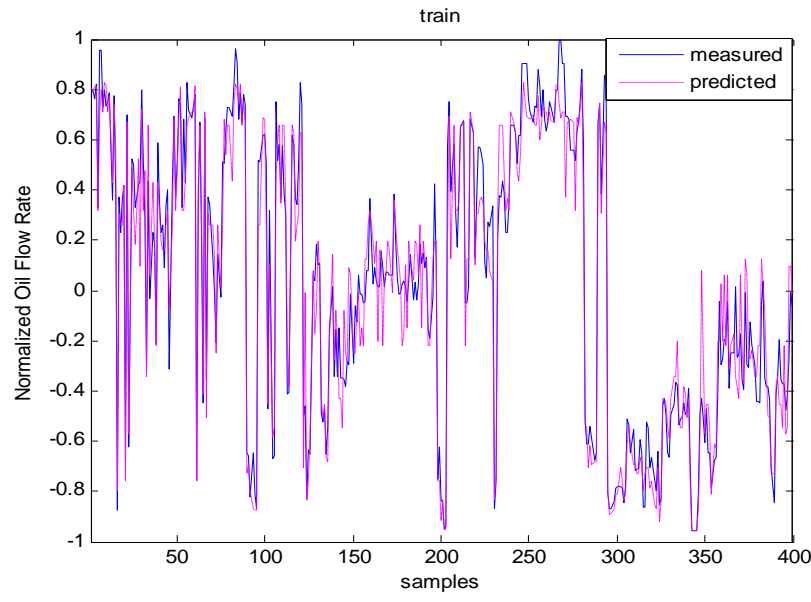

Fig 2: Comparison between measured and predicted oil flow rate, (ICA-ANN) Training. 


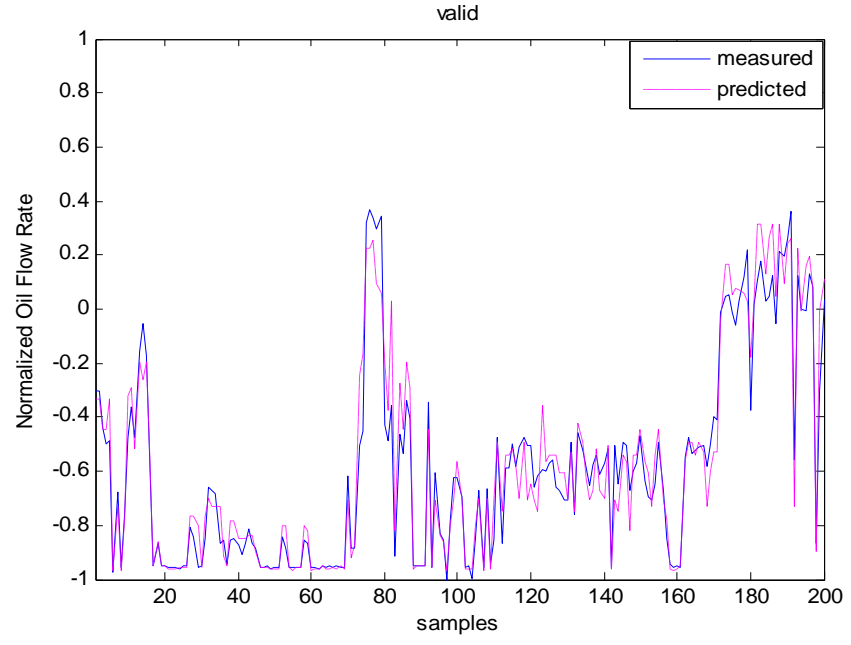

Fig 3: Comparison between measured and predicted oil flow rate, (ICA-ANN) validation phase

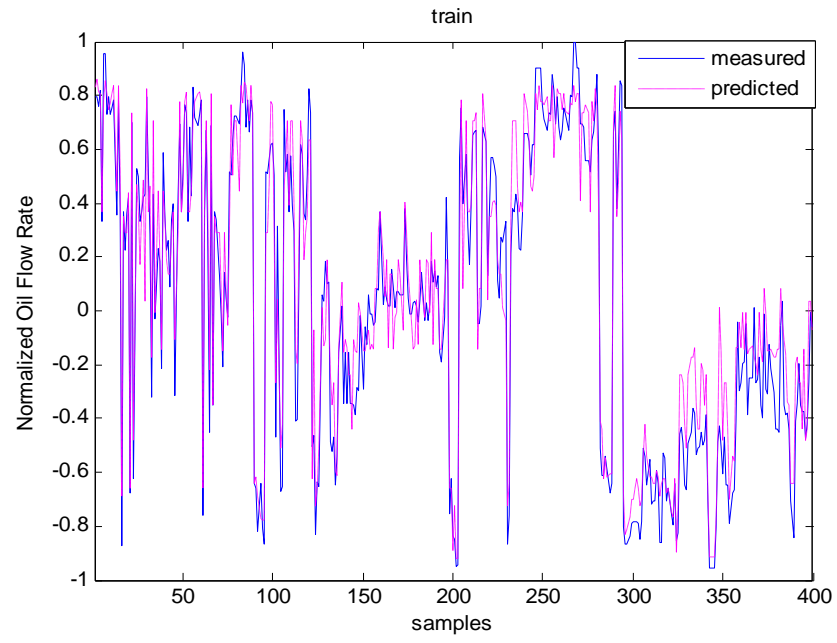

Fig 4: Comparison between measured and predicted oil flow rate, $(\mathrm{ANN})$ Training phase

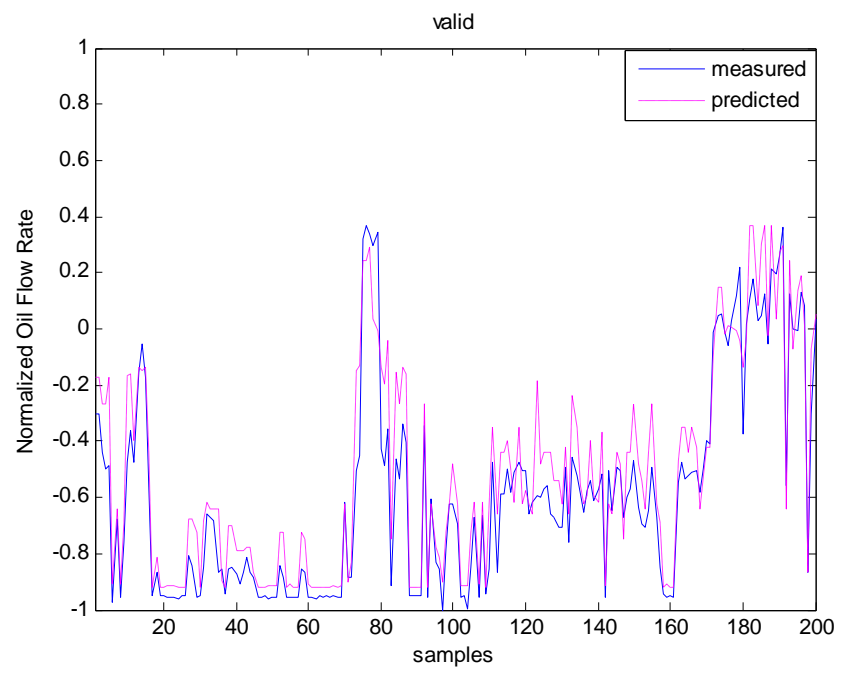

Fig 5: Comparison between measured and predicted oil flow rate, (ANN) validation phase

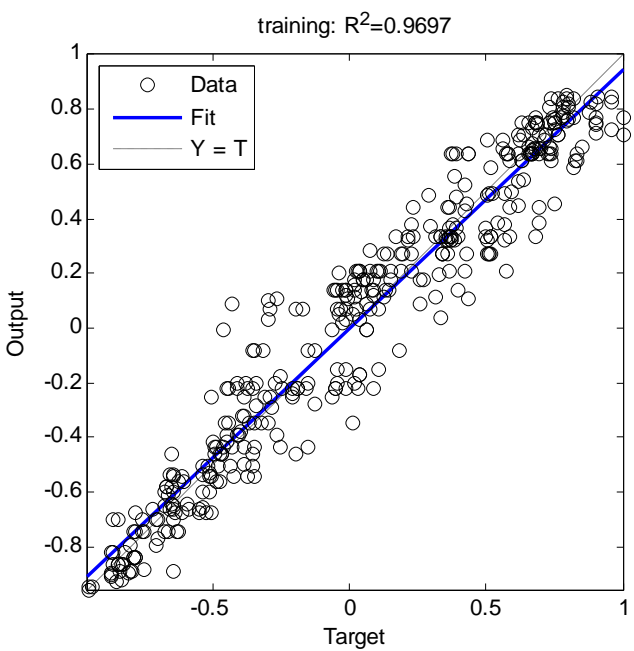

Fig 6: $\boldsymbol{R}^{2}$ ICA-ANN, Training phase

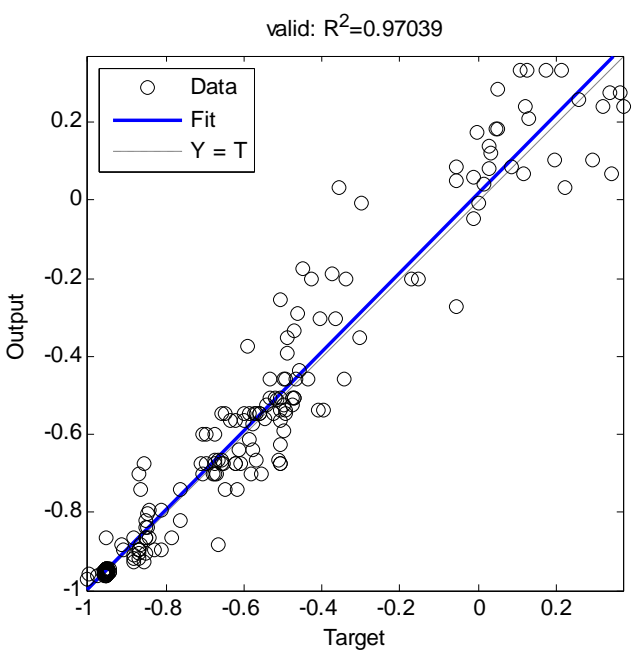

Fig 7: $\boldsymbol{R}^{\mathbf{2}}$ ICA-ANN, Validation phase

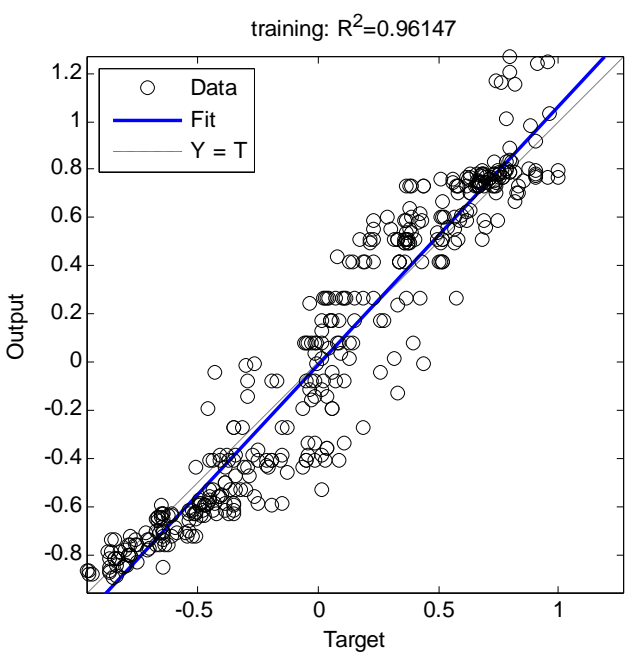

Fig 8: $R^{2}$ ANN, Training phase 


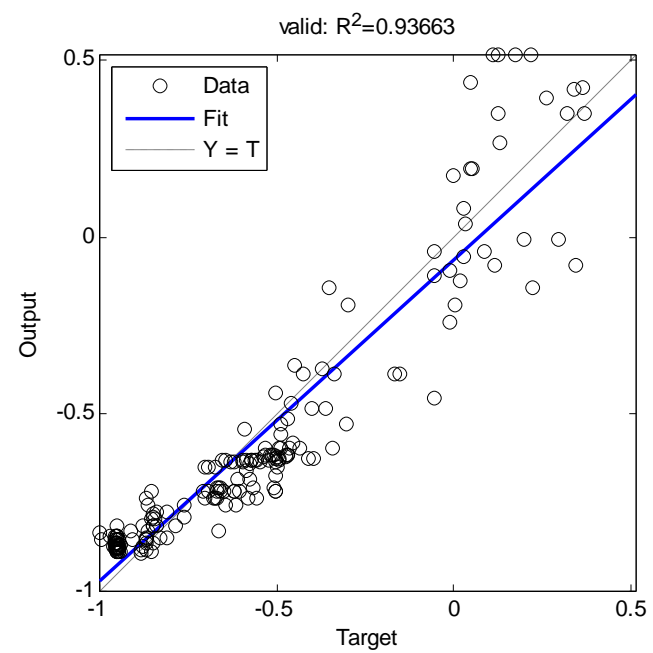

Fig 9: $\boldsymbol{R}^{2}$ ANN, Validation phase

Table 1. Comparison between the performances of ICAANN and ANN

\begin{tabular}{|c|c|c|}
\hline & ICA-ANN & ANN \\
\hline MSE & 0.0123 & 0.04702 \\
\hline $\boldsymbol{R}^{\mathbf{2}}$ & 0.9703 & 0.9366 \\
\hline
\end{tabular}

\section{CONCLUSION}

In this paper, we designed a model by using artificial neural network based on imperialist competitive algorithm for predict oil flow rate of the wells. Imperialist Competitive Algorithm (ICA) uses an evolutionary algorithm in order to optimize the initial weights of a feed forward neural network. The predictive performance of the proposed model (ICA-ANN soft sensor) is better than that of the traditional BP neural network; because of ICA-ANN combines local and global searching ability of the back propagation and ICA, respectively. By our purposed network, the requirement for extra piping and MFM eliminates, and flow rates of the wells reliably and continuously is monitored. Our proposed network has not radioactive source, so is safe for person and the environment, and can be used in place of the well.

A problem in ANN modeling is the determination of the optimal neural network topology. In this paper, ANN structure was determined manually. Our future work will be applied the Imperialist competitive algorithm for neural network structure optimization.

\section{REFERENCES}

[1] Rallo, R., Ferre-Gin, J., Arenas, A., \& Giralt, F. 2002. Neural virtual sensor for the inferential prediction of product quality from process variables. Computers and Chemical Engineering, 26, 1735-1754.

[2] Qu1, X., Feng, J. and Sun, W. 2008. Parallel Genetic Algorithm Model Based on AHP and Neural Networks for Enterprise Comprehensive Business, IEEE Intl. Conf. on Intelligent Information Hiding and Multimedia Signal Processing, pp.897-900.

[3] Souto, M.C.P.de, Yamazaki, A. and Ludernir, T.B. 2002. Optimization of neural network weights and architecture for odor recognition using simulated annealing, Proc. 2002 Intl. Joint Conf. on Neural Networks, Vol.1, pp.547-552.

[4] Reed, R. 1993. Pruning algorithms-a survey, IEEE Trans. Neural Networks Vol.4, pp.740-747.

[5] zhang, C., Shao, H. and Li, Y. 2000. Particle swarm optimization for evolving artificial neural network, 2000 IEEE Intl. Conf. on Systems, Man, and Cybernetics, Vol. 4, Oct. pp. $2487-2490$.

[6] Atashpaz-Gargari, E. Lucas, C. 2007. Imperialist Competitive Algorithm: An algorithm for optimization inspired by imperialistic competition. IEEE Congress on Evolutionary Computation, pp.4661-4667.

[7] Biabangard-Oskouyi, A., Atashpaz-Gargari, E., Soltani, N., Lucas, C. 2008. Application of Imperialist Competitive Algorithm for materials property characterization from sharp indentation test. To be appeared in the International Journal of Engineering Simulation.

[8] Rajabioun, R., Hashemzadeh, F., Atashpaz-Gargari, E., Mesgari, B., Rajaei Salmasi, F. 2008. Identification of a MIMO evaporator and its decentralized PID controller tuning using Colonial Competitive Algorithm. Accepted to be presented in IFAC World Congress.

[9] Atashpaz-Gargari, E., Hashemzadeh, F., Rajabioun, R. and Lucas, C. 2008. Colonial Competitive Algorithm, a novel approach for PID controller design in MIMO distillation column process, International Journal of Intelligent Computing and Cybernetics, 1 (3), 337-355.

[10] Sepehri Rad, H., Lucas, C. 2008. Application of Imperialistic Competition Algorithm in Recommender Systems. In: 13th Int'l CSI Computer Conference (CSICC'08), Kish Island, Iran.

[11] Hornik, K. Stinchcombe, M. and White, H. 1990. Universal approximation of an unknown mapping and its derivatives using multilayer feed forward networks. Neural Netw, 3(5):551-60.

[12] Hornick, K. Stinchcombe, M. and White, H.1989. Multilayer feed-forward networks are universal approximators. Neural Networks, 2, 359-366.

[13] Nash, J. E. Sutcliffe, J. V. 1970. River flow forecasting through conceptual models I: A discussion of principles. Journal of Hydrology, 10, 282-290. 